\title{
Status Quo of the Will of Chinese Professional Volleyball Players
}

\author{
Juan $\mathrm{Li}^{*}$ and Huan Kang
}

Institute of P.E, China West Normal University. Nan Chong 637009, Sichuan, China

\begin{abstract}
The survey on the willpower of Chinese professional volleyball players shows that Chinese female volleyball players are more tenacious than male players, while male players are better in self-control. Willpower of volleyball players is independent with training duration and individual skill level of the players. The ace spiker is more clear in target than the setter and auxiliary setter; the setter is more confident than the auxiliary setter; the auxiliary setter is more resilient than the ace spiker and blocker; and the libero player is more resilient than the ace spiker, blocker, and setter, and more decisive than the ace spiker and setter. In this case, proper training and competition cognitive orientation is recommended to be established, with focus on the differences of players in gender, position, skill, and psychological quality. Perfect willpower qualities can be developed, including improving compression resistance intensity by training, and increasing difficulty tolerance by competition.
\end{abstract}

Keywords: Player, sports will, volleyball.

\section{INTRODUCTION}

Will refers to the mental process to achieve the desired goals through conscious control and regulation of behavior in overcoming various difficulties [1]. Willpower of players the stable characteristics they manifested during will activities in sports training, competition, and life, with certain tendency [2]. As a dominant antagonistic sport, volleyball requires strong willpower and self-control of the players. The constant changing of offense and defense during the game requires the players to focus and ready to transfer their attention according to the rapidly changing situation in the field, and to decisively choose tactics to cooperate delicately and tacitly with their teammates, based on multiple information and individual skills. At the same time, the interference from schedule, venue, opponents, and spectators increase the demands to the willpower of the players. In the case that the tactics and skills of the high level players are close, the stable willpower plays a crucial role in depending the result of the competitions, especially in high level competitions. It is the strong willpower that determines the success of the players in overcoming the difficulties and resisting the pressure.

Psychologists has been keen to study the willpower in competitive sports, including practical qualitative research and empirical quantitative researches. The researches of Chinese experts are mostly concentrated on distinguishing and defining the dimensions of willpower, with measurement and analysis of some projects. As for the dimensions of willpower, Zhang Baohua raised six will quality for gymnasts: consciousness, decisiveness, courage, calmness, tenacity, and self-control [3]; Liang Chengmou, however, regarded the following as dimensions of willpower: toughness, tenacity, decisiveness, self-control, target definition, and conviction confirmation [2]. In terms of the measurement of will, Li Jing thinks the will of players varies from different sports; will qualities of different players in the same sports show no signigicant differences; comprehensive will levels of elite players are high in general [4]. The research of Hu Guiying shows that male players are more decisive than female players; there is no significant differences between amateur players and professional players; the comprehensive will qualities of teenage players, including selfcontrol, decisiveness, and toughness are significantly better than ordinary teenage studnets [5]. From the comparative research on the will quality of different exercise levels and genders, Wang Jing finds that male master players are more clear to the target than male first grade players, while first grade players are better than master players on tenacity; Female first grade players are better than second level players in toughness, decisiveness, and self-confidence and total score, with significant differences [6]. These inconsistent research results are caused by the different definitions and designs of scale, with few internal comparative to the will of players in a specific sport. Based on the characteristics of volleyball, this study adopts the will measurement scale of Liang Chengmou, to study if there are differences of will among high level volleyball players of different genders, positions, exercise levels, and exercise ages. Through questionnaires on will scores before competitions, non-experimental research method is adopted, from the angle of pre-competition psychology, to analyze the will of the players, so as to improve the operational level of will study. On this basis, active sports will can be established and developed, to improve the pre-competition mental state of Chinese high level players and promote normal functioning of sports skills. 


\section{SUBJECTS AND METHODS}

\subsection{Research Subjects}

Some team members who participated the 2010-2011 Chinese Super League Volleyball are selected as research subjects, with their will quality scales as research contents.

\subsection{Research Methods}

\subsubsection{Literature}

Over 30 articles on will and volleyball competition psychology were reviewed.

\subsubsection{Questionnair}

The "Will Scale of High Level Players" designed by Liang Chengmou was adopted, using seven level marking system (in which, 1 = totally noncompliance, 7 = fully compliant). The scale defines the structure of will quality as six dimensions of 38 questions, i.e. 1) toughness, 2) tenacity, 3) decisiveness, 4) self-control, 5) target definition, 6) selfconfidence. At the same time, validity $(\mathrm{r}=0.67, \mathrm{p}<0.05)$ and the test results (toughness 0.59 , self-control 0.67 , decisiveness 0.62 , tenacity 0.53 , self-confidence 0.57 , and target definition 0.48 ) of the internal consistency of the dimensions (Cronbach alpha) test results should meet the requirements [2].

\subsubsection{Mathematical Statistics}

The data was collected and processed using SPSS 13.0 for Windows XP software, with specific methods including t-test and ANOVA analysis.

\subsection{Test Methods}

The players, as a group, received individual test one day before the competition by the investigator, without interference from each other. The questionnaires were sent out and collected on the spot. In the 270 questionnaires, 264 were returned, with the rate of $97.8 \%$; among which, there were 254 valid questionnaires, with effective rate of $94.4 \%$

\section{RESULTS AND ANALYSIS}

Scores of each group were tested with frequency analysis, with the result of homogeneity and normal distribution of data from each group.

\subsection{General Condition of Will Quality of Chinese Profes- sional Volleyball Player}

Among the 254 valid questionnaires, 130 are from male players and 124 are from female players. Refer to Table 1 for statistics of other positions and scores of six dimensions. Compared with the high level volleyball players in Tian Ying's study, Chinese high-level volleyball players in this study show higher toughness $(\mathrm{M}=27.76$, Std. $=6.07)$, while lower tenacity $(\mathrm{M}=40.88$, Std. $=6.05)$, decisiveness $(\mathrm{M}=$ 29.62, Std. $=4.56)$, self-control $(\mathrm{M}=31.00$, Std. $=4.38)$, confidence $(\mathrm{M}=26.81$, Std. $=4.53)$, and target definition $(\mathrm{M}$ $=24.94$, Std lower. $=2.54)$, with significant difference [7]. The inconsistent results are related with different measurement environment and research subjects.

\subsection{Will Differences of Professional Volleyball Players}

\subsubsection{Will Differences of Professional Volleyball Players in Different Genders}

In order to examine the differences of volleyball players in different genders, the author conducted independent $\mathrm{T}$ test to the data, with the result (Table 2) showing volleyball players in different genders manifested heterogeneity of variance in decisiveness and target definition. After homogeneity test, female players are tougher than male players, with significant difference $(\mathrm{P}<0.05)$, while male players are better in self-control, with significant difference $(\mathrm{P}<0.05)$. There was no significant differences in toughness and selfconfidence.

Table 1. Descriptive statistics of scores in each dimensions of volleyball players.

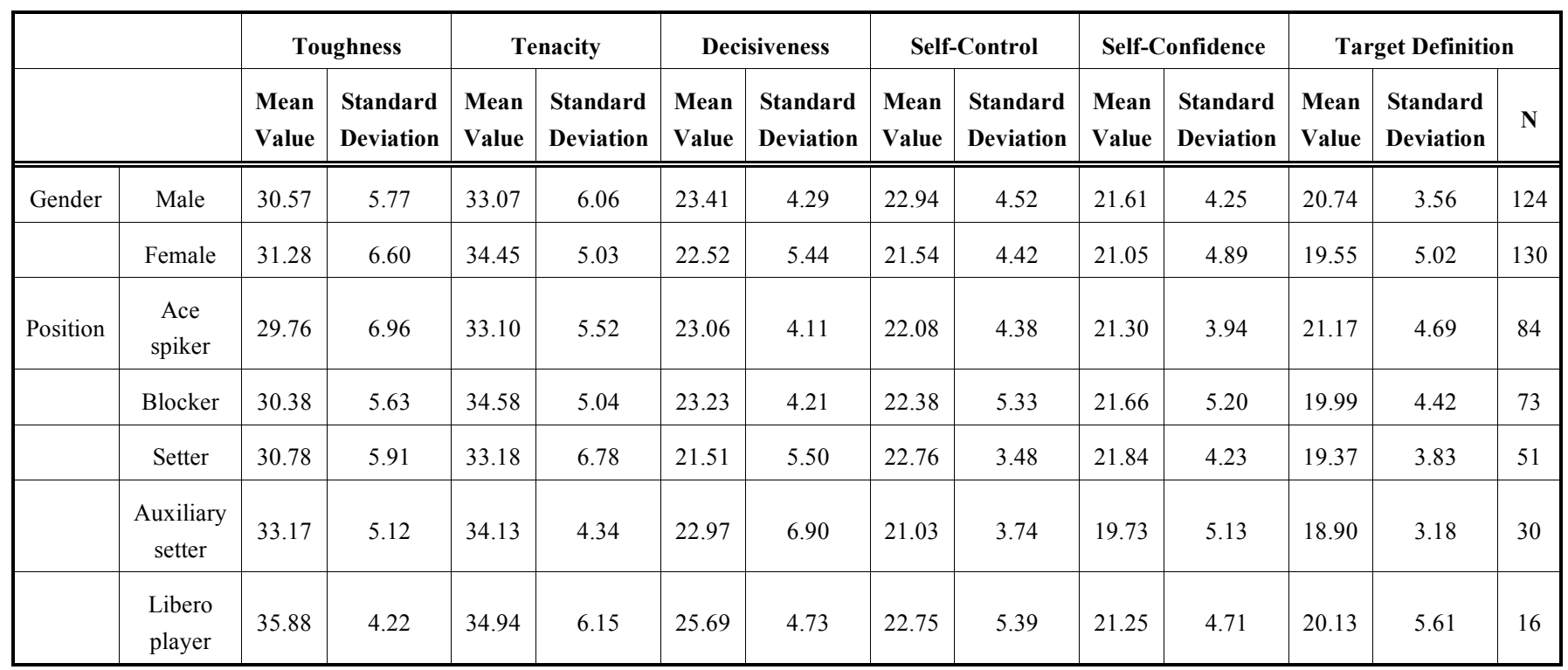


Table 2. Independent sample $T$ test for volleyball players in different genders.

\begin{tabular}{|c|c|c|c|c|c|c|}
\hline & & $\mathbf{F}$ & Sig. & $\mathbf{t}$ & df & Sig. (Bilateral) \\
\hline Toughness & Assuming the heterogeneity of variance & & & -.906 & 250.136 & .366 \\
\hline \multirow[t]{2}{*}{ Decisiveness } & Assuming the homogeneity of variance & 7.993 & .005 & 1.452 & 252 & .148 \\
\hline & Assuming the heterogeneity of variance & & & 1.460 & 243.424 & .146 \\
\hline Self-control & Assuming the homogeneity of variance & 1.113 & .292 & 2.505 & 252 & $.013 *$ \\
\hline Self-confidence & Assuming the heterogeneity of variance & & & .987 & 249.888 & .324 \\
\hline \multirow[t]{2}{*}{ Target definition } & Assuming the homogeneity of variance & 21.720 & .000 & 2.166 & 252 & .031 \\
\hline & Assuming the heterogeneity of variance & & & 2.184 & 232.907 & .030 \\
\hline
\end{tabular}

\subsubsection{Will Differences of Volleyball Players with Different Sports Level and Sports Ages}

Taking different sports levels (master level, national first grade level, and national second grade level) and different sports ages (1-5 years, 5-10 years, and over 10 years) as the dependent variable, the author conducted comparative research to the difference of will. The result shows that the sports will of players in different sports ages and sports levels has no significant differences in each dimensions; the sports will of volleyball players has nothing to do with the training duration and sports skills.

\subsubsection{Will Differences of Professional Volleyball Players in Different Positions}

In order to eliminate the gender implications, the author takes gender as covariate, and the position as dependent variable, to conduct multiple comparison and test to the will differences of players in different positions. The results are shown in the Table 3. There are significant differences among will of players in different positions in toughness, decisiveness, self-confidence, and target definition. Among which, there is significant difference in toughness between auxiliary setter and ace spiker and blocker, with $\mathrm{P}<0.05$; the

Table 3. Multiple comparison of the will of volleyball players in different positions.

\begin{tabular}{|c|c|c|c|c|c|}
\hline & $\mathbf{I}$ & $\mathbf{J}$ & Mean Difference & Standard Error & Significance \\
\hline \multirow[t]{4}{*}{ Toughness } & Auxiliary setter & Ace spiker & 3.405 & 1.291 & $.009 * *$ \\
\hline & & Blocker & 2.852 & 1.325 & $.032 *$ \\
\hline & Libero player & Ace spiker & 6.113 & 1.656 & $.000 * *$ \\
\hline & & Setter & 5.091 & 1.740 & $.004 * *$ \\
\hline \multirow[t]{2}{*}{ Decisiveness } & Libero player & Ace spiker & 2.628 & 1.334 & $.050 *$ \\
\hline & & Setter & 4.178 & 1.401 & $.003 * *$ \\
\hline Self-confidence & Setter & Auxiliary setter & 2.110 & 2.110 & $.047 *$ \\
\hline
\end{tabular}


toughness score of libero player is higher than ace spiker, blocker, and setter, with significant difference $(\mathrm{P}<0.01)$. For decisiveness, libero player scored higher than ace spiker, with significant difference $(\mathrm{P}<0.05)$, at the same time, libero player scored higher than setter, with significant difference $(\mathrm{P}<0.01)$. Setter scored higher in self-confidence than auxiliary setter, with significant difference $(\mathrm{P}<0.05)$. Ace spiker scored higher than setter and auxiliary setter in target definition, with significant difference $(\mathrm{P}<0.05)$. Will differences in players of different positions are related with the different requirements to players of different positions.

\section{DISCUSSION}

\subsection{Will Differences of Professional Volleyball Players in Different Genders}

The results showed that, among Chinese high level volleyball players, female players are tougher than male players, while male players are better in self-control than female players. Chinese female volleyball team took the lead as a glory for China and earned a lot of honor since the 1980s, which is inseparable from the toughness and tenacity of Chinese female volleyball players. Since the founding of PRC, Chinese female who have long been confined finally get liberation. Upon receiving equal social status, Chinese female players, with hard-working quality, went through training under limited conditions, with sports load in high strength and special acuteness. All of these require the spirit of overcoming difficulties and hard working. Chinese female volleyball players, under the spirit of "three requirements and heavy load", overcame the difficulties and objective conditions. After hard working of several generations, Chinese female players got the ability to won the Olympic gold medal and world champion. Correspondingly, the development of female volleyball also put forward higher requirements for the will quality of the players. With taller players, higher comprehensive physical quality, and greater tactical skills, the level of players from various countries are closer. In this case, female volleyball presents the features of large number of rebounding balls, strong confrontation, and fast pace of defense and offense conversion. During the game, the continuous ball keeps players in high attention to judge, take position, break the defense, and face the offense. These intense scene requires players to persist, overcome mental fatigue and emotional stress, and present competitive level with strong will. Compared with female volleyball, Chinese male volleyball started late. However, with superb selfcontrol, male volleyball players, based on their characteristics and rules of volleyball, creatively invented tactics of position difference and space difference, which lead the trend of world volleyball. Chinese male volleyball team contributed to the rich technical and tactical skills of volleyball, with promoting the spatial and in-depth development. The rapid development of female volleyball brought height and speed of volleyball to the extreme. The developing technical and tactical skills require the players, with years of training, to learn the superior self-control ability and adapt to the development of technical and tactical skills. High strength and fast-paced promotes the training requirements of players to comprehensively grasp the technical movements and adapt the changing situation; at the same time, players are required to overcome external and internal difficulties and weary emotion to develop better self-regulation ability.

\subsection{Will Differences of Volleyball Players with Different Sports Level, Sports Ages}

Willpower is essential for the players of various competitive sports. The long-term and periodic training requires players to overcome difficulties and insist on training. The fierce competition and highly intensive spirit raise high demands for the technical skills, fitness, and fighting spirit of players. Therefore, strong will is an important psychological guarantee for outstanding play. It is generally believed that the will of high level player is better than ordinary players. Results show, however, the will quality of volleyball players has nothing to do with the training duration and sports skills. This may be related to different focus of attention. The research on short track speed skaters by Wang Chengmin shows that, international male master players are better in target definition than Chinese male master players, while Chinese male master players are more tenacious. Female first grade players are better than second level players in toughness, decisiveness, and self-confidence and total score, with significant differences [8]. Studies also show that low level players tend to be more concerned about their achievements in one particular game, and show their own abilities to defeat others and achieve more scores, to prove themselves. High level players are more concerned if they can give full play to the level in training, to control the game, and to improve personal abilities [9]. The author believe that the excellent will quality of high level players manifests on the consistency of cognition to training and competition, and the execution of technical and tactical ability in the filed. Elite players can be strict with themselves during training to be clear and correct to each movement; and give full play to their abilities in training and implement the technical and tactical skills of the coach. On the contrary, due to lack of the relationship between training and competition, low level players are unable to produce the awareness of continuation of definition, and thus lowered the requirements to themselves. In same sports team, players with fewer sports ages succeeded less, with less presentation of their ability and value. Some players have playing experience, but mostly are failure. Over time, these athletes attributed their failure to lack of ability, and affect the rest of team to succeed. Therefore, they show poor quality in training and petrifying in competition. Some of them even face anxiety to resist competition. Despite the differences in situations, the coping method selected by players, mature or not, seem to be able to help ease the conflict and relieve the intense, but the consequential psychology can be positive or negative. For players with infirm willpower, positive coping methods, such as single defensive exercise, should be used in training to face difficulties actively, which will be more conducive to the development of will quality.

\subsection{Will Differences of Professional Volleyball Players in Different Genders}

There are significant differences among will of players in different positions in toughness, decisiveness, selfconfidence, and target definition. The ace spiker is more clear in target than the setter and auxiliary setter; the setter 
is more confident than the auxiliary setter; the auxiliary setter is more resilient than the ace spiker and blocker; and the libero player is more resilient than the ace spiker, blocker, and setter, and more decisive than the ace spiker and setter. Modern volleyball team is characterized by increasingly clear division of position. The comprehensive requirements on players is based on clear division of position. The six players are in different positions with different roles. Ace spiker is the main offender of the team, who is responsible for crucial tasks, to break through in difficult circumstances, mainly for middle and far net, back row and hit attack. The ability of the ace spiker is the prerequisite of the team to receive great results [10]. Ace spiker needs to ensure flexible tactics in offense, and properly adjust when the tactics are failed or the setter is not in the position. Therefore, ace spiker have to face the defense of the rivals more directly. Moreover, in many circumstances, the move of the ace spiker determines the effectiveness of the offense. These circumstances in crucial moments strengthens the cognition to the game rules of the ace spiker, to always remind himself to be decisive and resolute in breaking the defense. The clear goal plays a positive role in the control and adjustment of behaviors. Compared with ace spiker, setter and auxiliary setter bear less pressure to score in offense, which leads to the difference in target definition. The setter is like a "converter" that convert the balls from first pass and defense to auxiliary and offensive balls facilitating the offense and confusing the block of the rivals. The diversified tactics of setters require superb sports level and full self-confidence, to serve as the team core and achieve tactical intentions. Steady performance of setter determines the quality and style of the overall offense. The important role requires setters to organize offense with full selfconfidence, and encourage teammates in adverse circumstances and timely recognize in winning circumstances. Besides the technical skills of setters, auxiliary setters should be equipped with offense and defense ability and flexible skills. Although the self-confidence is not as high as setters, auxiliary setters is required to be more tough with the multiple roles. Libero players are responsible for receive and set balls and back row defense. An excellent libero play can not only compensate the lack of offense of the offensive players, but also conducive to the role of players, which change the offensive and defensive imbalance on the field [11]. Back row defense is the second line in the defensive system, serving as the basis to less loss and more scores. It can be seen from the matches that the number of back row offense greatly exceeds the number of block, with the highest technical error rate. Therefore, defense, particularly libero player defense is the key to raise offense success rate and reduce loss of scores. The special circumstances of libero players require players to face great pressure and suffering within and without. In many situations, the great uncertainty cause dilemma, where libero players have to decide in a short time, even make a move in a moment. This the performance of tenacity and courage.

\section{CONCLUSION AND SUGGESTION}

\section{Conclusion}

The results showed that, among Chinese high level volleyball players, female players are tougher than male players, while male players are better in self-control than female players.Willpower of volleyball athletes is independent with training duration and individual skill level of the athletes. The ace spiker is more clear in target than the setter and auxiliary setter; the setter is more confident than the auxiliary setter; the auxiliary setter is more resilient than the ace spiker and blocker; and the libero player is more resilient than the ace spiker, blocker, and setter, and more decisive than the ace spiker and setter.

\section{Suggestion}

(1) Coaches of each sports team of all levels should pay attention to the development and training of willpower, guide the players to build positive sports will and enhance the toughness of male players and self-control of female players with gradually increased difficulty.

(2) Establish correct orientation of training and competition, and consider will quality development equal with serious training and competition attitude. Young players aims to compete in the normal functioning level, while experienced players should play a leading role to encourage the teammates with fighting spirit and promote the growth of young players.

(3) Based on the characteristics of volleyball, the training of player's will should start from practical matches and adopt different principles, considering the differences in position, sports skills, and physiological quality. Thus to maintain the level of the training, carry forward the fighting and cooperation spirit of players on the filed, improve pressure resistance through training, enhance tolerance through competitions, to promote the excellent will quality of the high level volleyball players and contribute to the development of Chinese volleyball cause.

\section{CONFLICT OF INTEREST}

The authors confirm that this article content has no conflict of interest.

\section{ACKNOWLEDGEMENTS}

Declared none.

\section{REFERENCES}

[1] C. Liang, General Principles of Psychology, Three Gorges Press, Beijing, China, 1996, pp. 152-154

[2] C. Liang, Q. Fu, and J. Yu, "BTL-YZ-1 design of will scale of high level players," Paper Volume of 2003 Annual Academic Meeting of Beijing Psychological Association, 2003.

[3] B. Zhang, "Will quality of gymnast and its development," Shanghai Physical Education Institute, vol. 25, no. 5, pp. 133-134, 2010.

[4] J. Li, "Quantitative research on general will quality of players in different sports," Xi'an Physical Education Institute, no. 8, pp. 3742, 1991.

[5] G. Hu, and B. Xu, "Research on willpower of young players in Zhejiang province," Sports Sciences, vol. 21, no. 6, pp. 61-64, 1999.

[6] J. Yu, and Y. Cui, "Comparative research of will quality between track and field players with different sports levels and genders,' Academic Journal of Beijing Physical Education University, vol. 28, no. 6, pp. 761-763, 2005.

[7] Y. Tian, Y. Xu, and B. Li, "Comparative research on the will quality of skill-dominant players of different sports," Academic Journal of Wuhan Physical Education Institute, vol. 43, no. 7, pp. 52-55, 2009. 
[8] C. Wang, "Will quality compare of short track speed skating players of different genders and sports levels," Physical Education Journal, vol. 15, no. 11, pp. 96-99, 2008.

[9] X. Chen, "Research on achievement goal orientation and precompetition emotion of university players," Shandong Sports Science and Technology, vol. 24, no. 1, pp. 37-41, 2002.
[10] C. Ge, Latest Volleyball Training Theory and Practice, Beijing Physical Education University Press, Beijing, 2003, pp. 45-47.

[11] N. Li, and Z. Chen, "Current condition and evaluation of physical form of Chinese junior female volleyball players," China Sport Science and Technology, vol. 42, no. 5, pp. 89-91, 2006.

Received: June 10, 2015

Revised: July 29, 2015

Accepted: August 15, 2015

(C) Li and Kang; Licensee Bentham Open

This is an open access article licensed under the terms of the Creative Commons Attribution Non-Commercial License (http://creativecommons.org/licenses/by-nc/3.0/) which permits unrestricted, non-commercial use, distribution and reproduction in any medium, provided the work is properly cited. 